\title{
Réinscrire les études sur le journalisme dans une sociologie générale
}

Re-situating studies on journalism within general sociology

Reinscribir los estudios sobre el periodismo en una sociología general

Benjamin Ferron, Jean-Baptiste Comby, Karim Souanef et Jérôme

Berthaut

\section{CpenEdition}

\section{Journals}

Édition électronique

URL : http://journals.openedition.org/bssg/259

DOI : $10.4000 /$ bssg.259

ISSN : 2490-9424

Éditeur

Presses universitaires de Vincennes

Référence électronique

Benjamin Ferron, Jean-Baptiste Comby, Karim Souanef et Jérôme Berthaut, « Réinscrire les études sur le journalisme dans une sociologie générale », Biens Symboliques / Symbolic Goods [En ligne], 21 2018, mis en ligne le 12 avril 2018, consulté le 04 mars 2021. URL : http://journals.openedition.org/ bssg/259; DOI : https://doi.org/10.4000/bssg.259 


\title{
Réinscrire les études sur le journalisme dans une sociologie générale
}

\author{
Benjamin Ferron | Jean-Baptiste Comby | Karim Souanef | Jérôme Berthaut
}

La recherche française contemporaine sur le journalisme suit deux tendances principales qui méritent d'être interrogées d'un point de vue sociologique. D'une part, elle s'institutionnalise sur le modèle des journalism studies aux États-Unis (revues, groupement de chercheurs, etc.). Cette tendance à l'hyperspécialisation ne fait-elle pas courir le risque de déconnecter de processus sociologiques plus généraux les connaissances produites et les questionnements élaborés sur cet espace spécifique ? D'autre part, en raison du poids des sciences de l'information et de communication dans ce domaine de recherche et du développement des outils numériques de traitement de données, on assiste à un usage routinier d'analyses de contenus, peu ajustées aux exigences du métier de sociologue à partir du moment où elles s'autonomisent des méthodes principales (entretiens, observations, questionnaires, etc.) qui explorent les propriétés sociales, les trajectoires, les pratiques et représentations collectives. Or, les études qui cloisonnent «le journalisme » ou le réduisent à une affaire de mots, d'images ou de récits sont problématiques pour le sociologue lorsqu'elles ignorent les structures sociales au sein desquelles agissent les journalistes, les relations d'interdépendances entre l'espace de production et les autres champs, ou la manière dont ces rapports codéterminent ce qui est dit ou écrit par les journalistes et in fine lu, vu ou entendu par leurs publics (Champagne 1990).

C'est dans ce contexte que nous plaidons ici pour une réinscription des études sur le journalisme dans une sociologie plus générale. Et l'un des meilleurs moyens d'y parvenir nous paraît être d'enquêter en utilisant les instruments épistémologiques que sont les concepts de champ, d'habitus et de capital. L'articulation de ces concepts reste plus que jamais pertinente pour concilier les différents niveaux d'analyse (Bourdieu 2015), pour réaliser la dialectique entre le général et le particulier, entre les structures et les discours. Cette 
voie, empruntée depuis le début des années 1990 (Accardo 1995, 1998 ; Actes de la recherche en sciences sociales 1994, 2000 ; Bourdieu 1996 ; Champagne 1990 ; Duval 2004 ; Marchetti 1997, 1998, 2010 ; Marchetti \& Ruellan 2001 ; Neveu 1997, 2001 ; Pinto 2007), donne accès aux logiques de production, de diffusion et de réception de catégorisations du monde qui perpétuent l'ordre des choses ou ne le contestent qu'à la marge. Ce programme, qui vise moins l'accumulation de connaissances spécialisées que la mise à l'épreuve d'une théorie générale du social, a donné lieu récemment à de nombreux travaux qui gagnent à être mutualisés pour atteindre cet objectif (Sociétés contemporaines 2017 ; Comby 2017 ; Bouron [à paraître] ; Juhem \& Sedel 2016 ; Marchetti \& Souanef 2017 ; Souanef [à paraître] ; Nollet \& Desrumeaux [à paraître] ; Benson, Hesserus, Sedel [à paraître]). C'est pour fédérer ces recherches que se sont tenues, les 6 et 7 juin 2017, les journées de travail du réseau thématique 37 "Sociologie des médias » de l'Association française de sociologie, intitulées "Quelle autonomie (de la sociologie) du champ journalistique aujourd'hui ? » (voir le programme complet).

Dans la première partie de cet article, qui rend compte des principaux résultats présentés lors de ces journées de travail, nous montrerons que l'un des enjeux heuristiques de ces analyses à la fois structurales et constructivistes est de penser le journalisme comme partie prenante des rapports de pouvoir entre les classes sociales. Les travaux discutés ont en effet permis de saisir conjointement les logiques propres au journalisme et sa " localisation structurale »(Benson 1999) dans l'espace social global, c'est-à-dire les relations structurées entre le champ journalistique et d'autres champs, et ainsi de réinsérer les données empiriques accumulées (statistiques, entretiens, observations ethnographiques, questionnaires, corpus...) dans une sociologie générale de la production des biens symboliques. Poursuivant cette perspective, la seconde partie de ce texte traitera de la manière dont l'étude sociologique du journalisme peut contribuer à une compréhension de mécanismes sociaux plus généraux, et doit tenir compte de ces derniers. Est ainsi proposé un programme de recherche dont l'ambition est triple : cartographier le champ journalistique français contemporain ; penser les effets de sa subordination aux principes de l'économie financiarisée sur son fonctionnement et sur sa contribution à la perpétuation de l'ordre établi ; analyser les luttes de légitimité dont les journalistes et les médias, y compris les plus hétérodoxes d'entre eux, sont à la fois les enjeux et les agents.

\section{L'intégration accrue du champ journalistique au champ du pouvoir}

Si, lors de ces journées de juin 2017 , nous souhaitions faire le point sur l'état du champ journalistique contemporain, les recherches présentées ont mis au jour moins des bouleversements qu'une accentuation des logiques de structuration de cet espace. Le champ journalistique reste fortement subordonné aux principes de classement du champ politique mais il est de plus en plus organisé conformément aux catégories dominantes du champ économique. L'homogénéisation accrue de sa morphologie sociale dans sa fraction dominante, c'est-à-dire la moindre présence de membres issus des strates sociales intermédiaires et populaires, renforce un processus plus large de monopolisation des espaces conventionnels du débat public par certaines composantes des classes privilégiées (pour un exemple sur les terrains sanitaires ou écologiques (voir Henry 2007, Grossetête 2012 et Comby 2015). 


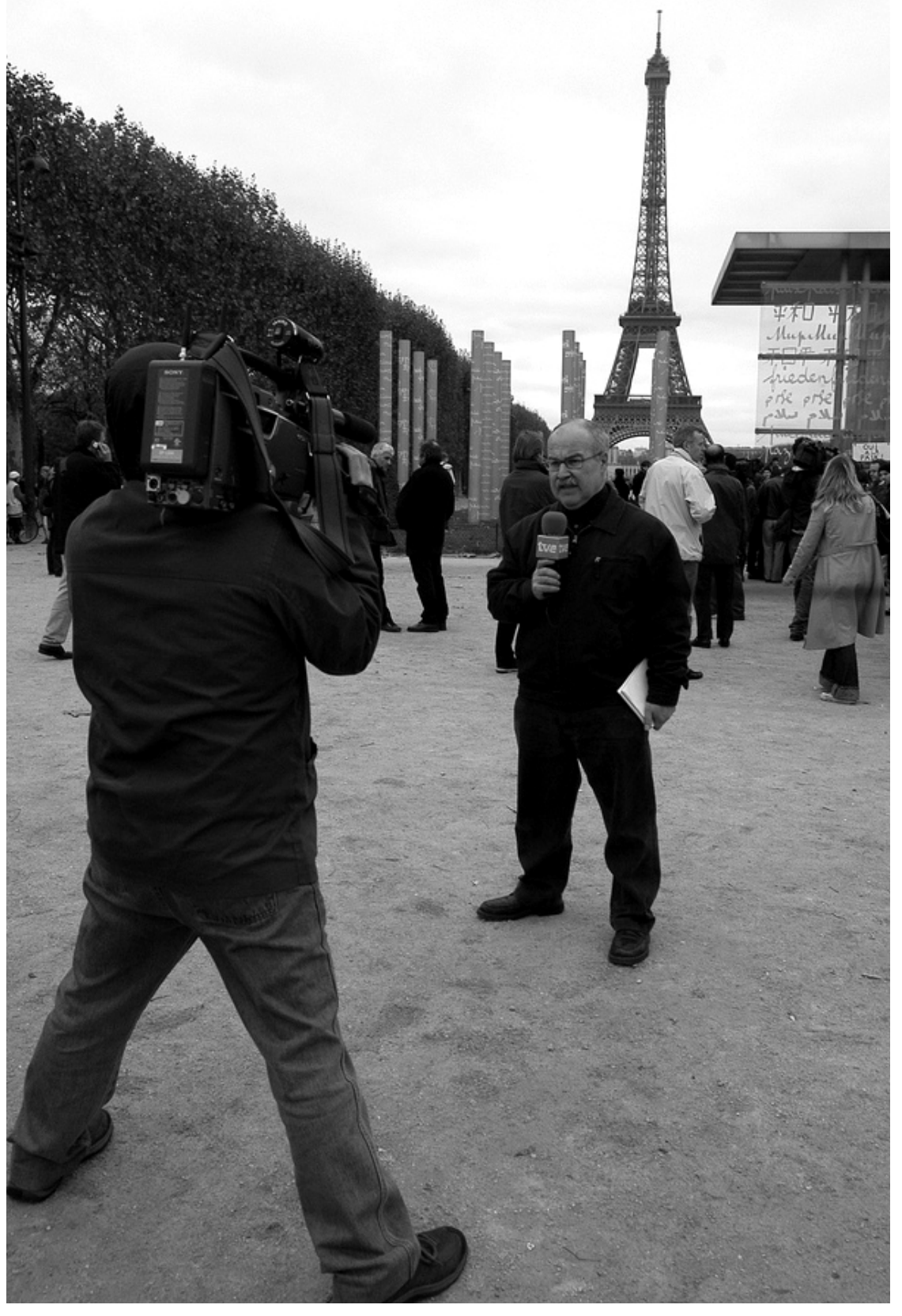

Figure 1. Reporters devant le mur de la paix

Suite à des épisodes d'affrontements entre la police et des habitants de banlieue populaire en région parisienne, des reporters de télévision font un plateau en prenant soin de cadrer la tour Eiffel, symbole de la nation française (20 novembre 2005).

Source : Wikimedia Commons (CC BY-SA 2.5)
La place croissante de la théorie des champs dans les recherches internationales sur le journalisme

La première journée a été inaugurée par une conférence de Rodney Benson consacrée à la genèse, à l'essor et à la récente institutionnalisation d'une sociologie du journalisme ancrée dans la théorie des champs au sein des media studies aux EtatsUnis et, au-delà, en Europe et en Asie. Quelques années après la traduction en anglais de travaux de Pierre Bourdieu sur les champs de production culturelle (1993) et le champ journalistique (1998), I'Université de Berkeley (États-Unis) accueille, en 2000, une rencontre entre des chercheurs français membres ou proches du Centre de sociologie européenne (Eric Darras, Julien Duval, Dominique Marchetti, Erik Neveu, Loïc Wacquant) et des chercheurs états-uniens (Rodney Benson, Eric Klinenberg, Michael Schudson, Daniel Hallin, Timothy Cook, Ted Glasser, Neil Fligstein). Leurs échanges sont à l'origine du livre Bourdieu and the Journalistic Field (Benson \& Neveu 2005). L'ouvrage constitue un tournant dans la reconnaissance internationale de ce courant de la sociologie. II est recensé dans des revues scientifiques internationales de référence, cité plus de 2000 fois en douze ans ${ }^{1}$ et traduit en chinois en 2017.

Cette publication prend place dans un mouvement plus large de multiplication des recherches mobilisant la théorie des champs pour analyser l'emprise croisée du journalisme et des champs politique, économique ou intellectuel (Couldry 2003 ; Davis \& Seymour 2010; Krause 2011 ; Phelan 2008 ; Ryfe 2016 ; Shin 2016) ; les mécanismes de reproduction et les transformations partielles des routines professionnelles dans des contextes de changements des modèles entrepreneuriaux et technologiques, en particulier avec le

1 Source : Google Scholar. 
développement des supports numériques (Lewis 2012 ; Powers \& Vera-Zambrano 2016 ; Tandoc 2014) ; les sous-champs spécialisés du journalisme comme le photojournalisme, le journalisme de voyage ou le journalisme d'art (Solaroli 2016 ; Botma 2015 ; Rosenkranz 2016) ; des comparaisons internationales entre pays occidentaux et/ou non occidentaux (Christin 2014 ; Handley \& Rutigliano 2012 ; Hovden 2012 ; Mellor 2007) ; les conditions sociales de la circulation transnationale des modèles journalistiques (Kuipers 2011 ; Christin 2016) ; le rôle de l'habitus dans les relations entre les journalistes et leurs sources (Nash 2016) ; ou encore les processus différenciés de structuration des circuits de production et de diffusion des informations en fonction des positions des agents dans les rapports sociaux de domination (Lewis 2012, Ryfe 2016). Ces travaux ont donné lieu à des débats productifs (Couldry 2007), notamment avec le courant néo-institutionnaliste sur la question du rôle de l'État dans l'organisation des champs journalistiques nationaux, une question peu traitée dans les écrits de Bourdieu (Benson 2006). Ils ont également conduit en 2017 à des sessions spéciales consacrées à la théorie des champs lors des congrès de I'International Communication Association et de l'American Sociological Association. Cette conférence introductive a ainsi donné l'occasion aux chercheurs français de mesurer, en contraste avec le peu de considération dont jouit paradoxalement cette approche en France aujourd'hui, l'ampleur et la diversité des avancées scientifiques permises par l'application de la théorie des champs à l'étude du journalisme, tant sur les plans empirique que méthodologique et théorique.

\section{Caractériser l'interdépendance}

\section{entre les champs journalistique, politique et économique}

Suite à cela, la première journée qui portait sur « la double dépendance " (Champagne 2016), à savoir sur les relations entre le journalisme et les champs politique et économique, s'est poursuivie par deux sessions de travail. Discutée par Annie Collovald, la première session explorait l'emprise réciproque entre les univers journalistique et politique. Rappelant les fondements de cette dépendance et ses évolutions, cette session interrogeait également, à partir de l'analyse de trajectoires sociales d'agents en politique, le poids des ressources médiatiques dans le jeu politique et, par ce biais, la place du journalisme dans les transformations du champ politique. Dominique Marchetti a ainsi présenté ses recherches sur les correspondants étrangers et les journalistes des agences de presse ainsi que sur la production et la circulation transnationale de l'information au Maroc (Hivert \& Marchetti 2015). Ce pays est caractérisé par le contrôle étroit du champ politique sur le champ journalistique national ainsi que sur les correspondants étrangers et les journalistes travaillant pour des agences de presse. Éric Darras a montré pour sa part que les transformations du champ politique français qui accompagnent la montée en puissance de « nouveaux médias " (chaînes d'information en continu, supports en ligne) tendent largement à reproduire l'ordre social qu'ils sont censés révolutionner : sans nier les possibilités accrues pour certains acteurs politiques de second rang d'accéder à une plus grande visibilité, l'exposition médiatique des professionnel·le.s de la politique reste largement corrélée à leur position institutionnelle (Darras 1995, 2017). Le concept de « capital médiatique » des agents politiques et son articulation avec celui de champ a enfin été questionné et précisé par Jérémie Nollet, coorganisateur de journées d'étude sur ce sujet en septembre 2016 à l'Institut d'études politiques de Toulouse ${ }^{2}$. Proposé par Patrick Champagne en 1990 dans Faire l'opinion, dans la continuité des travaux de

2 Journées d'étude «Un capital médiatique ? Usages et légitimation de la médiatisation en politique », organisée par Compol-AFSP, Sciences-Po Toulouse, septembre 2016. 
Pierre Bourdieu sur le champ politique, en vue d'analyser les capacités inégales des groupes sociaux à « faire l'événement » aux yeux des journalistes (Champagne 1990 : 237-245), le concept de " capital médiatique " désigne " une forme de capital spécifique, qui permet d'accéder et d'agir à des degrés divers - et selon les périodes parfois - dans le champ journalistique » (Marchetti 1998 : 278 ; voir aussi Couldry 2003 ; Davis \& Seymour 2010). Jérémie Nollet est revenu sur le débat existant avec d'autres chercheurs craignant que ce concept n'ajoute rien au répertoire analytique des sciences sociales (capitaux économique, social, culturel, symbolique) qui n'ont pas besoin, pour reprendre la formule d'Erik Neveu, «d'accumuler les capitaux » (2013).

La deuxième session, discutée par Paul Lagneau-Ymonet, envisageait les formes de l'emprise du marché sur le journalisme et en particulier les agents sociaux qui l'exercent, à commencer par les dirigeants de presse d'information politique et générale en France étudiés par Julie Sedel. À partir des premiers résultats d'une habilitation à diriger les recherches en cours, cette dernière a souligné l'hétérogénéité de leurs positions de pouvoir en faisant le lien avec leurs trajectoires sociales et professionnelles (Sedel, en cours). Au-delà du seul marché de l'information, les échanges ont porté sur la formation au métier de journaliste, et sur la manière dont les investissements patronaux l'infléchissent. Dans cette perspective, Ivan Chupin a ainsi analysé le processus de « mise au pas gestionnaire " de l'univers des formations au journalisme à la fin des années 1990, à travers le cas du Centre de formation et de perfectionnement des journalistes (Chupin 2008). En prenant comme exemple les nominations dans l'audiovisuel extérieur, Romain Lecler a proposé d'envisager la télévision non pas comme un espace uniquement structuré par le capital économique, mais comme un lieu également réglé par des logiques hautement politiques incarnées par les trajectoires des agents occupant des positions de pouvoir dans l'audiovisuel public (Lecler 2015, 2016). Ces derniers se répartissent en effet autour de deux interprétations rivales de l'audiovisuel extérieur : l'une de gauche, plus diplomatique et culturelle, autour de TV5 ; l'autre de droite, centrée sur la France et l'information continue, autour de France 24 et son « ancêtre " Canal France International.

Deux interventions transversales ont clôturé cette première journée. Celle de Julien Duval a traité des possibilités offertes par la méthode de l'analyse des correspondances multiples dans l'étude d'un sousespace (la presse économique) afin d'appréhender statistiquement le poids relatif et la position des agents dans l'ensemble du champ journalistique (Duval 2013). Patrick Champagne est revenu, quant à lui, sur les critiques dont le journalisme est régulièrement l'objet, et sur les spécificités du champ journalistique qui s'est historiquement construit à l'intersection de trois champs aux logiques partiellement incompatibles : les champs économique, politique et intellectuel (Champagne 2006, 2016).

\section{Logiques du champ journalistique, rapports de classes et luttes spécifiques}

La deuxième journée interrogeait plus précisément la structuration interne du journalisme et la contestation (relative) des règles en vigueur dans cet espace. La troisième session, discutée par Lise Bernard, examinait la morphologie de la population des journalistes établie à partir de leurs trajectoires sociales, de leurs formations et de leurs parcours professionnels. Les contributions sont revenues sur la genèse du champ journalistique et l'état incertain de ses frontières tout en cherchant à caractériser ses principes de division interne. Que nous disent de l'autonomie du champ journalistique les données disponibles sur les professionnel.le·s de l'information et leurs carrières ? Comment caractériser les coordonnées sociales, 
les orientations politiques et les styles de vie des journalistes et, partant, la place du journalisme dans les rapports entre les classes sociales ? Olivier Baisnée a présenté une recherche en cours fondée sur une prosopographie des hommes de presse français à la fin du $\mathrm{XIX}^{\mathrm{e}}$ siècle, réalisée à partir de l'Annuaire de la presse française. Évitant le piège téléologique consistant à considérer comme une nécessité historique la constitution, dès 1880, d'un champ professionnel relativement autonome, qui ne sera avérée que dans le premier tiers du $x x^{e}$ siècle, l'auteur étudie l'hétéronomie constitutive d'un espace social auquel les agents, multipositionnés, ne s'identifient pas entièrement. Samuel Bouron a montré que les transformations de l'espace des écoles spécialisées dans la formation de journalistes correspondaient aux transformations du champ journalistique. Dans ce but, il a mobilisé des données non seulement sur les modes de sélection et de formation des étudiant.e.s, en particulier celles et ceux issu.e.s des classes supérieures, mais aussi sur les différents modes d'insertion sur le marché du travail ou les principales oppositions dans les styles de vie des diplômé.e·s (Bouron 2014). À partir d'une enquête longitudinale par questionnaire et entretiens auprès d'étudiant $\cdot e \cdot s$ en écoles « reconnues » de journalisme, Géraud Lafarge a analysé les conditions sociales de possibilité de l'entrée dans la profession de journaliste, ainsi que celles du maintien dans cet espace et l'accès inégal aux différentes positions qu'il offre en fonction des origines sociales des candidat.e.s (Lafarge \& Marchetti 2011, 2017). Enfin, Antoine Machut a traité des conditions de maintien et de sortie des journalistes dans la presse financière, à partir d'une enquête statistique et d'entretiens portant sur leur carrière : il a ainsi pointé combien l'état des marchés financiers conditionnait le développement de cette spécialité et « le fort pouvoir d'attraction du monde de la finance sur ces individus " (Machut, [en cours]).
La quatrième session explorait les mécanismes sociaux qui sous-tendent les entreprises de contestation de la légitimité journalistique. Une sociologie du champ journalistique attentive aux spécificités de cet espace et des sous-espaces qui le composent permet de rendre compte finement des conditions sociales dans lesquelles des agents en contestent les logiques dominantes de consécration. Ces individus et ces groupes ontils des propriétés, notamment en termes de capital économique, social et culturel, qui les prédisposeraient à s'engager dans des luttes pour la transformation des rapports de forces prévalant dans le champ journalistique ? Parmi ceux-ci, lesquels ont les moyens de convertir des ressources externes pour peser davantage dans les luttes internes ? Lesquels privilégient la construction d'espaces de contestation relativement protégés de la domination symbolique exercée par les agents mieux dotés en capitaux spécifiques ? Discutées par Benjamin Ferron, les communications ont porté tout d'abord sur les rapports entre l'univers des petites maisons d'édition critique et la presse, analysés par Sophie Noël. Celle-ci a ainsi montré leurs relations paradoxales aux médias : ces maisons d'édition espèrent toujours, en définitive, qu'on parlera de leurs publications, bien qu'elles récusent le plus souvent cette aspiration à la certification médiatique (Noël 2010, 2012). Les échanges ont ensuite porté sur les propriétés sociales et les pratiques des journalistes bloggeurs politiques analysées par Marie Neihouser. Critiquant la doxa démocratique qui accompagne l'essor de ces espaces numériques censés favoriser l'arrivée de nouveaux·elles entrant.e.s dans le champ (journalistes amateurs, citoyen.ne.s...), l'auteure montre au contraire, à partir d'une base de données exhaustive (plus de 3500 blogs), que les effets de ces espaces numériques sont très largement dépendants de la position des agents dans ou vis-à-vis du champ professionnel (Neihouser 2014, 2015). La session s'est terminée par une interrogation sur les conditions et les effets de la contestation des logiques dominantes 
du champ journalistique, à travers une analyse de l'histoire et du fonctionnement de l'association Acrimed, fondée en 1996 et spécialisée dans une critique " intransigeante et informée 》 des médias, présentée sous forme d'autoanalyse par Julien Salingue qui en fut l'un des salariés et rédacteurs pendant plusieurs années. À l'issue de ces journées, se dessine donc un champ journalistique français toujours plus homogène à son pôle dominant - avec une porosité plus forte entre les pôles dits commerciaux et intellectuels - mais aussi davantage intégré au champ du pouvoir qu'il y a vingt ans. Sa double dépendance, vis-à-vis des champs économique et politique, ne cesse de se renforcer et avec elle, le rétrécissement du spectre des visions médiatiquement légitimes du monde social. Cette situation ouvre ainsi la voie à de multiples entreprises de subversion des règles du jeu imposées par cette structure oligopolistique. Si Internet a pu faire évoluer certaines manières de mettre en forme ces visions, il n'a pas réellement modifié les structures sociales du champ journalistique. Celles-ci continuent de s'ajuster aux attentes de l'ordre établi. D'où l'enjeu accru des espaces " alternatifs" du journalisme au sein desquels progressent des modes non conventionnels de traitement de l'actualité et des problèmes sociaux. Les mutations du champ journalistique, ces vingt dernières années, se sont donc sans doute limitées à ses marges.

\section{Cartographier le champ journalistique contemporain français}

Ces constats, provisoires, encouragent surtout à poursuivre le décloisonnement des études sur le journalisme pour les inscrire dans une sociologie générale. Cartographier le champ journalistique contemporain en France nous semble dès lors constituer un programme de recherche pouvant y contribuer. Tout en se nourrissant de travaux tels que ceux précédemment présentés, un tel projet permettrait, au niveau empirique, de situer historiquement et socialement le journalisme pour mieux le penser relationnellement (par exemple comme une composante du champ du pouvoir) et pour mieux saisir comment il travaille (et est travaillé par) les hiérarchies matérielles, symboliques et institutionnelles entre les groupes sociaux. Au niveau théorique, cette perspective aiderait à le questionner à partir de concepts qui ne lui sont pas spécifiques pour comprendre de quelles manières des processus sociaux plus larges opèrent et/ou se jouent sur le terrain journalistique (par exemple des logiques de méconnaissance sociale, de naturalisation, de stigmatisation ou de célébration). Pareille entreprise scientifique impliquerait notamment un travail de mutualisation de données collectées sur différents terrains, que ce soit à partir d'un découpage par supports (presse écrite, radio, télévision, web) ou par spécialités (politique, économie, sport, culture, fait divers, etc.). L'enjeu serait donc aussi institutionnel et relèverait d'une realpolitik de la recherche. II s'agirait en effet de dégager des marges d'autonomie pour se donner le temps d'enquêter dans une perspective théorique qui n'est pas toujours au goût des tenants de la doxa managériale dominant actuellement la recherche ${ }^{3}$. Le réseau thématique sociologie des médias de l'Association française de sociologie nous semble pouvoir être le lieu à partir duquel seraient impulsées dans la durée des recherches articulées à cette cartographie. II a vocation à être un espace de coordination et de travail réunissant des sociologues qui étudient le journalisme ou d'autres espaces ou activités sociales liés aux médias. À ce jour, nous souhaiterions initier, ou remettre sur le métier, trois chantiers qui nous semblent particulièrement pertinents au regard de l'état des recherches établi lors des journées de juin.

3 Comme semble d'ailleurs l'indiquer le récent déclassement de sociologues au concours d'entrée du CNRS par la direction de l'établissement (Beaud, Broussard, Pudal, Topalov 2017) 


\section{Morphologie sociale}

Le premier entend renseigner plus systématiquement les coordonnées sociales, les styles de vie, les goûts culturels, les orientations politiques et l'économie des sociabilités des journalistes. Comment se positionnent-ils/elles dans l'espace social et politique ? Comment ces positions sociales s'articulentelles avec leur répartition au sein des différents médias ? Quelles socialisations secondaires peut-on observer au sein des métiers du journalisme ? II s'agirait en effet de mieux comprendre comment, en fonction des expériences socialisatrices constitutives de leur habitus, ils/elles élaborent des croyances professionnelles et adhèrent aux logiques engendrant leur subordination aux classements politiques et aux impératifs commerciaux. Cette caractérisation morphologique de l'espace des journalistes semble également nécessaire pour mieux comprendre les visions du monde qu'ils/elles diffusent, mais aussi pour replacer dans un espace hiérarchisé les enquêtes ethnographiques et localisées sur leurs pratiques professionnelles.

S'il apparaît que les principes de structuration du champ s'accentuent, la recherche manque cependant encore de données quantitatives permettant de préciser ces lignes de clivage internes et externes. Le niveau d'entrée pourrait être celui des rédactions à propos desquelles diverses informations seraient collectées : organigramme, place occupée dans l'entreprise de presse, niveaux de revenus des salarié $\cdot e \cdot s$, formation et parcours professionnels, etc. Si se trouve confirmée l'hypothèse de l'homogénéisation sociale des journalistes sorti.e.s d'école et disposé·e.s à occuper des positions stables, voire dominantes dans le champ, alors étudier le " haut du panier » journalistique reviendrait avant tout à travailler sur les fractions culturelles des classes supérieures et au-delà sur les franges dominées du champ du pouvoir.
Réinscrire ainsi le champ journalistique dans une analyse de la structure d'ensemble de l'espace social peut alors aider à comprendre ce qui se joue dans les modes de consommation des offres informationnelles. Les médias peuvent en effet être pensés comme une instance de socialisation des individus, aux côtés toutefois d'autres instances génératrices de dispositions plus consistantes comme la famille, l'école ou la sphère professionnelle. Or la moindre diversité sociale des journalistes (sous le double poids de leur professionnalisation et de la rationalisation des stratégies d'accès à cet espace de valorisation symbolique) ne tend-elle pas à favoriser la circulation des visions du monde conformes aux intérêts et valeurs des classes dominantes? Les publics des médias seraient ainsi plus disposés à adhérer à ces hiérarchisations médiatiques des enjeux sociaux, des options politiques, des goûts culturels et au-delà des conduites individuelles. En retour, l'homogénéisation de l'offre informationnelle estampillée « journalisme professionnel » et son ajustement aux dispositions des classes supérieures et des fractions privilégiées des classes moyennes, ne favoriseraient-elles pas l'essor et la captation d'autres publics par des producteurs de biens symboliques considérés comme hybrides, diffusés par des canaux moins spécialisés (portails, réseaux sociaux, plateformes de partages...) ?

\section{Emprise du champ économique}

La seconde dimension de la structuration du champ journalistique sur laquelle il paraît pertinent de porter la focale concerne sa dépendance aux logiques économiques. Dans une économie de plus en plus financiarisée et libéralisée, comment les logiques commerciales continuent-elles de se déployer au sein du champ journalistique ? Par quels types de passeurs les rationalités économiques sont-elles relayées et comment sont-elles retraduites pour peser concrètement sur lefonctionnementdes entreprises de presse, par exempleàtravers 
une division du travail et des personnels dédiés (régies publicitaires, services commerciaux et évènementiels, directions de production), mais aussi des consultant e.s (Brandewinder 2009) ? Dans quelle mesure les habitus des journalistes-dirigeant.e.s d'entreprises de presse et de leurs sous-traitants évoluent-ils sous l'effet du renforcement de la rationalité marchande ? Quels sont les effets de cette dernière sur les contenus médiatiques, illustrés par le cas emblématique du journalisme sportif (Marchetti \& Souanef 2017) ? Mettre au jour les modalités ordinaires de l'imbrication entre le champ économique et le champ journalistique revient donc à s'intéresser plus spécifiquement à la manière dont les agents et institutions (multi)positionnés à la frontière de différents champs, par la diversification de leurs activités (notamment médiatiques), leur rôle d'intermédiaires, et/ou la diversité des ressources accumulées, parviennent à se hisser dans le champ du pouvoir et à renforcer leur position. On pense notamment aux conglomérats et dirigeants des grandes firmes (de l'industrie, des BTP, des télécommunications, de la finance...) et à leurs investissements dans le domaine des médias. Les propriétés sociales et les trajectoires des dirigeant·e.s de médias et d'organisations parties prenantes des univers médiatiques (chaînes, groupes de presse, sociétés de production, maisons d'édition, associations et syndicats professionnels, etc.), ainsi que leurs relations et espaces de sociabilités avec les autres composantes du champ du pouvoir constituent des terrains d'étude susceptibles d'expliquer la place des entreprises de presse dans l'organisation de ce dernier.

\section{Hétérodoxies journalistiques}

Le troisième chantier est une sociologie de la construction du rôle des médias et du journalisme comme problème public. Le dynamisme de la quatrième session des journées de travail a montré toute la pertinence de ce questionnement sur les marges du champ, les modes de dénonciation des règles établies, les fondements sociaux de l'hétérodoxie qui sont autant de rappels de ce qu'est le fonctionnement routinier du champ et de son emprise sur les pratiques médiatiques. Comment saisir, à l'instar d'un travail mené sur le cas turc (Yavcan \& Ongur 2016), les conditions sociales de genèse et de déploiement des critiques du champ journalistique, c'est-à-dire de la transformation des organes de presse, de leur fonctionnement et de leurs agents en des enjeux de mobilisations, de controverses publiques et d'action gouvernementale (Neveu 2015) ? Quelles conditions sociales favorisent ce déplacement de problématique à travers lequel des médias d'information et des journalistes passent du statut d'intermédiaires dans la construction des problèmes publics à celui d'objets de débats publics, pouvant déboucher sur des luttes dans le champ politique et la transformation du cadre légal et institutionnel qui définit leur place et leur rôle dans l'espace social global ?

Ce chantier entend faire dialoguer des traditions de recherche que la division contemporaine du travail scientifique tend à séparer. Ainsi, la sociologie de l'action publique s'est penchée depuis une vingtaine d'années sur la prise en compte des contraintes médiatiques dans les processus décisionnels (Nollet 2010), mais a laissé largement en friche la question du rôle de l'action publique dans la structuration des systèmes médiatiques et des espaces professionnels qui le composent (Hallin \& Mancini 2004). De même, la sociologie des mouvements sociaux a pris acte de l'importance croissante des médias dans la conduite de l'action collective protestataire (Réseaux 1999) et de la contribution des groupes d'intérêt militants à la définition des politiques publiques (Meyer, Jenness, Ingram 2005), mais peu de travaux sociologiques se sont intéressés aux media movements, ces « réseaux de citoyens et d'associations qui mènent des combats politiques pour réaliser des transformations sociales, en particulier en ce qui concerne la presse et les médias 
en général » (Waisbord 2009). Ensuite, peu de recherches en sociologie du journalisme et des médias se sont intéressées aux conditions sociales - différentes selon les champs pris en compte de production de la croyance dans les effets politiques des médias de masse sur les groupes sociaux contemporains (Desrumaux \& Nollet 2014). Enfin, les nombreuses recherches conduites depuis une vingtaine d'années dans le domaine des " médias alternatifs " (Downing 2010 ; Atton 2015) ont dans l'ensemble insuffisamment étudié les contraintes législatives, administratives et financières qui pèsent sur les médias dits alternatifs, citoyens ou radicaux ainsi que les propriétés et trajectoires sociales des « médiactivistes 》 (Cardon \& Granjon 2010). Ces dernières constituent pourtant des conditions sociales de l'autonomisation relative de ces agents au sein ou à l'égard des groupes dont ils émanent. Plusieurs terrains d'enquête mériteraient à ce titre d'être explorés au prisme de la théorie des champs, qu'il s'agisse d'analyser les mobilisations de journalistes, le travail de spécialisation de la communication militante, les luttes électorales ou législatives à propos des médias d'information ou encore les processus de différenciation et de structuration des espaces de la critique des médias et des médias " alternatifs " (Anderson 2015 ; Ferron 2015, 2016).

À l'instar des deux précédents, un tel chantier permettrait d'aborder des phénomènes qui dépassent les seules questions du journalisme et des médias. Dans quelle mesure l'analyse des recompositions à l'œuvre dans le champ journalistique permet-elle de saisir des processus comme les mutations du travail et des entreprises sous l'effet des politiques néolibérales, la dualité renforcée du système d'enseignement français entre les grandes écoles et les universités, l'émergence d'actions collectives orientées vers, ou ancrées dans, les logiques de la communication médiatique ou encore l'emprise de l'État sur la structuration des champs de production symbolique?
Ces trois chantiers impliquent d'enquêter dans le même temps sur la façon dont Internet a pu, ou non, transformer le champ journalistique. Alors que l'existence d'une "ère numérique " semble faire consensus, il est urgent de questionner ce que ce type de formulation présuppose souvent : l'existence d'un espace déconnecté du social et de ses régularités. Les rares travaux qui ont mobilisé une problématique et des méthodes sociologiques sur ces terrains convergent pourtant pour montrer l'importance des logiques de reproduction sociale à l'œuvre dans l'accès et les usages des dispositifs socio-numériques (Christin 2014 ; Neihouser 2014, 2015 ; Maratea 2008 ; Tandoc 2014 ; Maitra \& Tandoc 2017). En d'autres termes, il paraît indispensable d'étudier les conditions qui permettent aux rapports de force offline de se réfracter et se perpétuer dans les hiérarchies online, et plus rarement de subvertir les premières.

Opérateurs centraux de la production et de la circulation des visions légitimes du social, de ses enjeux comme de ses principes de classement, les professionnel.le.s de l'information sont au cœur des luttes auxquelles se livrent des agents sociaux pour asseoir leur légitimité, les rapports de force étant aussi des rapports de sens. Par conséquent, mettre au jour les inégalités d'accès aux définitions médiatiques des enjeux suppose bien souvent d'étudier diverses inégalités matérielles et symboliques entre des groupes aux intérêts politiques différents, voire antagonistes. Et ce d'autant plus que les journalistes occupent des positions d'interface entre différents domaines d'activité sur lesquels ils/elles jouissent d'une sorte de droit de regard et, en accordant du crédit à tel ou tel groupe, peuvent consolider, voire renforcer, la position que celuici occupe dans une hiérarchie socioprofessionnelle. En d'autres termes, le champ journalistique contribue à l'inégale distribution sociale des privilèges. II s'ensuit qu'à bien des égards l'ordre symbolique, c'est-à-dire la hiérarchie des di-visions du monde, est 
une expression transfigurée de l'ordre social. Réinscrire les études sur le journalisme dans une sociologie plus générale doit donc permettre de progresser dans cette compréhension des ressorts médiatiques de la perpétuation, par-delà les « nouveaux visages et les nouveaux usages », des structures sociales établies.

Benjamin Ferron

Université Paris-Est Créteil - Centre d'étude des discours, images, textes, écrits communication (CEDITEC)

Jean-Baptiste Comby

Université Paris 2 - Centre d'analyse et de recherche interdisciplinaire sur les médias (CARISM) et Centre nantais de sociologie (CENS)

Karim Souanef

Université de Lille - Centre d'études et de recherches administratives, politiques et sociales (Ceraps)

Jérôme Berthaut

Université de Bourgogne - Laboratoire communications, médiations. organisations, savoirs (CIMEOS)

\section{Références bibliographiques}

ACTES DE LA RECHERCHE EN SCIENCES SOCIALES (1994). “ L'emprise du journalisme », 101-102.

ACTES DE LA RECHERCHE EN SCIENCES SOCIALES (2000). « Le journalisme et l'économie », 131-132.

AcCARDO Alain (dir.) (1995). Journalistes au quotidien. Pour une socioanalyse des pratiques journalistiques. Bordeaux, Le Mascaret.

AccARDo Alain (dir.) (1998). Journalistes précaires. Bordeaux, Le Mascaret.
ANDERSON Benjamin (2015). «Alternative Media \& Bourdieu's Field Internal Resistance or External Competition ? ». Transnational Worlds of Power Journal, 1(1) : 177-189.

Atton Chris (2015). The Routledge Companion to Community and Alternative Media. Londres, Routledge.

Beaud Stéphane, Boussard Valérie, Pudal Romain, Topalov Christian (2017). « L'élimination des sociologues au concours du CNRS, symptôme du management autoritaire de la recherche en sciences sociales ». Zilsel. [En ligne] https://zilsel.hypotheses.org/2857\# ftn1 [consulté le 3 juillet 2017].

BEnson Rodney (1999). « Field Theory in Comparative Context : A New Paradigm for Media Studies ». Theory and Society, 28(3) : 463-498.

BENSON Rodney (2006). "News media as a "journalistic field" : What Bourdieu adds to new institutionalism, and vice versa ». Political Communication, 23(2) : 187-202.

Benson Rodney \& NeVEu Erik (dir.) (2005). Bourdieu and the Journalistic Field. Cambridge, Polity Press.

Benson Rodney, Hesserus Mattias, Sedel Julie (à paraître). How Press Ownership Matters. Oxford, Oxford University Press.

Botma Gabriel J. (2015). «Cultural Capital and the Distribution of the Sensible : Awarding Arts Journalism in South Africa (201-2014) 》. Journalism Studies, 18(2) : 211-227.

BouRdieu Pierre (1996). Sur la télévision. Paris, Liber.

BouRdieu Pierre (2015). Sociologie générale, volume 1. Cours au Collègue de France 1983-1985. Paris, Seuil. 
BouRon Samuel (2014). Apprendre à penser comme un journaliste. Construction sociale des catégories de connaissances professionnelles et division du travail journalistique (thèse de doctorat en sociologie). Amiens, Université de Picardie Jules Verne.

BRANDEWINDER Marie (2009). Le Journalisme et les consultants : le conseil médias dans les entreprises de presse (thèse de doctorat en science politique). Rennes, Université de Rennes.

Cardon Dominique \& Granjon Fabien (2010). Médiactivistes. Paris, Presses de SciencesPo.

Champagne Patrick (1990). Faire l'opinion. Le nouveau jeu politique. Paris, Minuit.

Champagne Patrick (2006). " À propos du champ journalistique ». Questions de communication, 10 : 197-210.

ChAmpagne Patrick (2016). La Double Dépendance. Sur le journalisme. Paris, Raisons d'agir.

CHRISTIN Angèle (2014). Clicks or Pulitzer? Web Journalists and their Work in the United States and France (thèse de doctorat en sciences de la société). Paris, EHESS/Princetown University.

CHRISTIN Angèle (2016). « Is Journalism a Transnational Field ? Asymmetrical Interactions and Symbolic Domination in Online News ». The Sociological Review. 64(2) : 212-234.

ChupIN Ivan (2008). Les Écoles du journalisme : les enjeux de la scolarisation d'une profession (1899-2008) (thèse de doctorat en science politique). Paris, Université Paris-Dauphine.

ComBY Jean-Baptiste (2015). La Question climatique. Genèse et dépolitisation d'un problème public. Paris, Raisons d'agir.
COMBY Jean-Baptiste (dir.) (2017). Enquêter sur l'internationalisation des biens médiatiques et culturels. Rennes, Presses Universitaires de Rennes (PUR).

Couldry Nick (2003). « Media Meta-Capital : Extending the Range of Bourdieu's Field Theory ». Theory and Society, 32(5-6) : 653-677.

CoUldRY Nick (2007). « Bourdieu and the Media : The Promise and Limits of Field Theory ». Theory and Society, 36(2) : 209-213.

DARRAS Éric (1995). "Le pouvoir "médiacratique" ? Les logiques du recrutement des invités politiques à la télévision ». Politix, 30 : 183-198.

DARRAS Éric (2017). « Introduction. Champ journalistique, ordre social et ordre politique ». Sociétés contemporaines, $106:$ 5-20.

DAVIS Aeron \& SeYmour Emily (2010). « Generating forms of media capital inside and outside a field : the strange case of David Cameron in the UK political field ». Media, Culture \& Society, 32(5) : 739-759.

DesRumaux Clément \& Nollet Jérémie (2014). «Présentation. Quelques apports, limites et dépassements des théories de la "mediatization" du politique ». Réseaux, $187:$ 9-21

DownING John D. H. (dir.) (2010). Encyclopedia of Social Movement Media. Thousand Oaks/Londres/New Delhi/Singapour, Sage Publications.

DUVAL Julien (2004). Critique de la raisonjournalistique. Lestransformations de la presse économique en France. Paris, Seuil.

DuVAL Julien (2013). « L'analyse des correspondances et la construction des champs ». Actes de la recherche en sciences sociales, $200: 110-123$.

FERRON Benjamin (2015). La communication internationale du zapatisme (1994-2006). Rennes, PUR. 
FERron Benjamin (2016). «Professionnaliser les « médias alternatifs «? Enjeux sociaux et politiques d'une mobilisation (1999-2016) ». Savoir/ Agir, 38 : 21-28.

Grossetête Mathieu (2012). Accidents de la route et inégalités sociales. Les morts, les médias et l'État. Bellecombe-en-Bauges, Le Croquant.

Hallin Daniel \& Mancini Paolo (2004). Comparing Media Systems. Three models of Media and Politics. Cambridge, Cambridge University Press.

HANDLEY Robert L. \& RUtigliano Lou (2012). « Journalistic field wars : defending and attacking the national narrative in a diversifying journalistic field ». Media, Culture \& Society, 34 (6) : 744-760.

HenRY Emmanuel (2007). Amiante : un scandale improbable. Sociologie d'un problème public. Rennes, Presses Universitaires de Rennes.

HIVERT Joseph \& MARCHETTI Dominique (2015). « Numériquement marginaux, mais politiquement importants ? La médiatisation internationale d'une association des droits de l'homme au Maroc ». Journal des anthropologues, 142-143 : 227-251.

Hovden Jan Fredrik (2012). "A Journalistic Cosmology. A Sketch of Some Social and Mental Structures of the Norwegian Journalistic Field ». Nordicom Review, 33 (2) : 57-76.

Juhem Philippe \& Sedel Julie (dir.) (2016). Agir par la parole. Porteparoles et asymétries de l'espace public. Rennes, Presses Universitaires de Rennes.

Krause Monika (2011). " Reporting and the transformations of the journalistic field : US news media, $1890-2000 »$. Media, Culture \& Society, 33 (1) : $89-104$.
KUIPERS Giselinde (2011). " Cultural globalization as the emergence of a transnational cultural field : transnational television and national media landscapes in four European countries ». American Behavioral Scientist, $55(5): 541-557$

Lafarge Géraud \& MARChetTI Dominique (2011). " Les portes fermées du journalisme. L'espace social des étudiants des formations 'reconnues'», Actes de la recherche en sciences sociales, 189 : 72-99.

Lafarge Géraud \& MarchettI Dominique (2017). « Les hiérarchies de l'information. Les légitimités « professionnelles » des étudiants en journalisme ». Sociétés contemporaines, $106: 21-46$.

LECLER Romain (2015). « Naissance et mort d'une direction aux Affaires étrangères. L'administration de l'audiovisuel extérieur au prisme de ses entrepreneurs en bureaucratie ». Politix, 112 : 197-222.

LECLER Romain (2016). " Une diplomatie audiovisuelle à la carte. Ce que les pratiques de recrutement révèlent de la « politique audiovisuelle extérieure " française ". Revue internationale de politique comparée, 23 (2) : 175-197.

LEWIS Seth (2012). « The tension between professional control and open participation : journalism and its boundaries ». Information, Communication \& Society, 15 (6) : 836-866.

MACHUT Antoine (en cours). Cadrage et Encastrement de la Finance. Mesurer et comprendre le changement de médiatisation des techniques financières en France et en Grande-Bretagne (thèse de doctorat en sociologie). Grenoble, Université Grenoble Alpes.

MAITRA Julian \& TANDOC Edson (2017). « News organizations' use of Native Videos on Facebook: Tweaking the journalistic field one algorithm change at a time ». New Media \& Society. [En ligne] http://journals.sagepub.com doi/abs/10.1177/1461444817702398 [consulté le 15 janvier 2018]. 
MARATEA Ray (2008). «The e-Rise and fall of social problems : the blogosphere as a public arena ». Social Problems, 55 (1) : 139-160.

MARCHETTI Dominique (1997). Contribution à une sociologie des transformations du champ journalistique dans les années 80 et 90 (thèse de doctorat en sociologie). Paris, EHESS.

MARCHETTI Dominique (1998). « Les conditions de réussite d'une mobilisation médiatique et ses limites. L'exemple d'Act Up-Paris ». In CurApp (dir.). La politique ailleurs. Paris, Presses Universitaires de France, 277-297.

MARCHETTI Dominique (2010). Quand la santé devient médiatique. Les logiques de production dans la presse. Grenoble, Presses Universitaires de Grenoble.

MARCHETTI Dominique \& RUELLAN Denis (2001). Devenir journalistes. Sociologie de l'entrée sur le marché du travail. Paris, La Documentation française.

MARCHETtI Dominique \& SOUANEF Karim (2017). « La médiatisation du football : un jeu sous contrôle. Les économies de la production de l'information sur les compétitions européennes en France », Pôle Sud. Revue de science politique de l'Europe méridionale, $47: 61-78$.

Mellor Noha (2007). Arab Journalism : Problems and Prospects. Édimbourg, Edinburgh University Press.

Meyer David S., Jenness Valerie, Ingram Helen (dir.) (2005). Routing the Opposition : Social Movements, Public Policy, and Democracy. Minneapolis, University of Minnesota Press.

NASH Chris (2016). «News Sense, Sources, Sociology and Journalism ». In NASH Chris (dir.). What is journalism ? The Art and Politics of a Rupture. New York, Palgrave MacMillan : 165-202.
NeIHOUSER Marie (2014). « Les blogs politiques, un support favorable à l'engagement citoyen ? ». Politiques de communication, 2 (3) : 59-84.

NeIHOUSER Marie (2015). Un nouvel espace médiatique ? Sociologie de la blogosphère politique française (thèse de doctorat en science politique). Montpellier, Université de Montpellier.

NEVEU Erik (1997). Une société de communication ?. Paris, Montchrestien.

NEVEU Erik (2001). Sociologie du journalisme. Paris, La Découverte.

NEVEU Erik (2013). « Les sciences sociales doivent-elles accumuler les capitaux ? À propos de Catherine Hakim, Erotic Capital, et de quelques marcottages intempestifs de la notion de capital ». Revue française de science politique, $63: 337-358$.

Neveu Erik (2015). Sociologie des problèmes publics. Paris, Armand Colin.

NoEl Sophie (2010). « Les petits éditeurs « critiques » et la presse : une relation ambigüe ». Communication et langages, $163:$ 29-46.

NOEL Sophie (2012). L'édition indépendante critique : engagements politiques et intellectuels. Villeurbanne, Presses de l'Enssib.

Nollet Jérémie (2010). Des décisions publiques « médiatiques 》? sociologie de l'emprise du journalisme sur les politiques de sécurité sanitaire des aliments (thèse de doctorat en science politique). Lille, Université Lille 2.

Nollet Jérémie \& DesRumeaux Nicolas (dir.) (à paraître). Ouvrage sur le concept de « capital médiatique ». Rennes, Presses Universitaires de Rennes. 
PINTO Eveline (dir.) (2007). Pour une analyse critique des médias. Le débat public en danger. Bellecombe-en Bauges, Le Croquant.

Phelan Sean (2008). " Democracy, the Academic field and the (New Zealand) journalistic habitus ». Studies in Language and Capitalism, 3-4 161-180.

Powers Matthew \& Vera-Zambrano Sandra (2016). « Explaining the Formation on Online News Startups in France and the United States : A Field Analysis ", Journal of Communication, 66 (5) : 857-877.

RÉSEAUX (1999). Médias et mouvements sociaux. 98.

RYFE David M. (2016). Journalism and the public. Keys concepts in journalism. Cambridge, Polity.

RosenKRANZ Tim (2016). "Becoming Entrepreneurial : Crisis, Ethics and Marketization in the Field of Travel Journalism ». Poetics, $54: 54-65$.

SEDEL Julie (en cours). « Sociologie d'une position patronale : dirigeant de média », HDR de sociologie, Université de Strasbourg.

SHIN Yongjun (2016). « Connecting Political Communication with Urban Politics : A Bourdieusian Framework ». International Journal of Communication, $10: 508-529$

SOCIÉTÉS CONTEMPORAINES (2017). Champ journalistique, ordre social et ordre politique. 106.

SOLAROLI Marco (2016). «The rules of a middle-brow art : Digital Production and Cultural Consecration in the Global Field of Professional Photojournalism ». Poetics, $59: 50-66$.

SOUANEF Karim (à paraitre). Journalistes sportifs. Sociologie d'une spécialité dominée. Rennes, Presses universitaires de Rennes.
TANDOC Edson C. Jr (2014). « Audiences, Journalists, and Forms of Capital in the Online Journalistic Field ». Romanian Journal of Communication and Public Relations, 16 (3) : 23-33.

WAISBORD Silvio (2009). "Bridging the Divide between the Press and Civic Society. Civic Media Advocacy as "Media Movement" in Latin America ". Nordicom Review. Jubilee Issue : 105-116.

Yavcan Basak \& ONGUR Hakan Ovunc (2016). « Determinants of Media Criticism in a Democracy in Transition : Applying Field Theory to Turkey ». International journal of communication, 10 : 2422-2441. 